\title{
Medium-Sized HCC: Achieving Effective Local Tumor Control with Combined Chemoebolization and Radiofrequency Ablation
}

\author{
Eleni Liapi, MD and Jean-Francois H. Geschwind, MD
}

Division of Vascular and Interventional Radiology, Russell H. Morgan Department of Radiology and Radiological Science, The Johns Hopkins Hospital, Baltimore, MD

Hepatocellular carcinoma (HCC) is the third most important cause of cancer-related mortality worldwide. ${ }^{1}$ In the USA, there are approximately 18,000 new HCC cases every year, and HCC incidence and mortality rates continue to increase, particularly among middle-aged Black, Hispanic, and White men. ${ }^{1}$ Most patients with HCC present with underlying liver cirrhosis, and therefore any treatment should be focused on these two disease entities concomitantly. Locoregional treatment options such as transcatheter arterial chemoembolization (TACE) and radiofrequency ablation (RFA) are minimally invasive treatment options that may individually or in combination address the pertinent issue of successful tumor targeting and preservation of liver function.

TACE involves intra-arterial injection of chemotherapy followed by embolization. Under this general definition of TACE, many variations in the application of this therapy exist, namely in the selection of chemotherapy and embolic material, as well as the technical component of injection (selective versus nonselective, occlusive versus nonocclusive, slow over time infusion versus standard injection). ${ }^{2}$ TACE is the most widely used therapy for intermediatestage HCC. ${ }^{3}$

Radiofrequency ablation (RFA) is a locoregional treatment option that induces thermal injury to the tissue through electromagnetic energy deposition. While a significant number of clinical series have reported curative outcomes of small HCCs $(<3 \mathrm{~cm})$ using RFA, published study data for treatment of larger tumors have been mixed. ${ }^{4,5}$ One way of increasing the volume of RF thermal lesions is to decrease the blood flow of the tumor area to be

(C) Society of Surgical Oncology 2011

Published Online: 23 March 2011

J.-F. H. Geschwind, MD

e-mail: jfg@jhmi.edu ablated with subsequent decrease in heat loss by convection. ${ }^{6}$ This may be achieved by combining TACE, which reduces arterial inflow to the tumor, with RFA. Several studies have demonstrated the synergistic cytotoxic effects of TACE with RFA. ${ }^{7-10}$

In this issue of Annals of Surgical Oncology, Kim et al. retrospectively evaluate and compare the effectiveness of TACE combined with RFA (TACE + RFA) against that of RFA alone in a selected group of patients with mediumsized $(3.1-5 \mathrm{~cm}) \mathrm{HCC}$. This retrospective study included 57 patients who were treated with TACE + RFA and 66 treated with RFA alone. The TACE protocol in the TACE + RFA group included selective catheterization of the relevant hepatic artery, infusion of cisplatin $(2 \mathrm{mg} / \mathrm{kg}$ body weight) into the hepatic artery for $15 \mathrm{~min}$, followed by infusion of an emulsion of iodized oil and cisplatin into a selected subsegmental feeding artery and embolization with 1-mm-diameter absorbable gelatin sponge particles until complete arterial flow stasis was observed. The RFA treatment was performed 3 days to 2 weeks after TACE in patients who underwent TACE. The RFA protocol included treatment with multiple overlapping insertions of a single electrode or an electrode cluster with a $3-$ or $3.5-\mathrm{cm}$ exposed tip, until complete ablation occurred, including both the visible tumor and margins of at least $0.5-1.0 \mathrm{~cm}$ in the surrounding hepatic parenchyma. Patients were followed up the day after treatment by contrast-enhanced computed tomography (CECT) of the liver for presence of residual tumor enhancement and possible complications. Patients with enhancement near the treated lesion underwent an additional session of RFA for the residual lesion. Treatment efficacy was evaluated by CECT 1 month after the procedure. Patients with completely ablated tumors and without evidence of new intrahepatic tumors were followed-up by CECT performed every $2-3$ months. Patients with new tumors in the ablated lesion or other new 
intrahepatic tumors during follow-up were treated with RFA if they met the criteria for RFA; patients who did not meet these criteria were treated with TACE. Patients were followed until death, time of surgical resection or liver transplantation, or the end of this study. Measureable clinical outcomes were major complications and local tumor progression. Major complications were low in both groups $(0 \%$ for the TACE + RFA and 3\% for the RFA group). However, local tumor control was significantly better in the TACE + RFA group $(P<0.001)$. The 1-, 3-, 5 -, and 7-year local tumor progression rates were significantly lower in the TACE + RFA group $(9 \%, 40 \%, 55 \%$, and $66 \%$, respectively) than in the RFA alone group ( $45 \%$, $76 \%, 86 \%$, and $89 \%$, respectively).

The patients in this study had single tumors, no evidence of vascular invasion or extrahepatic spread, and were mostly hepatitis B positive (65.9\%). Hepatitis B infection, even though still very common in Asia, is declining in Europe and the USA. Interestingly, in the USA, it is estimated that up to 4 million people have chronic hepatitis $\mathrm{C}$ virus $(\mathrm{HCV})$ infection, and subsequently, $11.7 \%$ of them will develop HCC. ${ }^{11}$ There was one significant difference between the two groups in terms of patient population demographics: Child-Pugh (CP) score was significantly higher (class B) in the RFA group. This may have inadvertently influenced the multivariate analysis performed in this study, indicating that CP class B and RFA alone were an independent prognostic factor influencing overall survival. The TACE protocol employed in this study is mostly used in Asia. ${ }^{12,13}$ In the USA, the most commonly used TACE protocol involves injection of doxorubicin, cisplatin, and mitomycin $\mathrm{C}$ mixed with iodized oil, followed by nonocclusive arterial embolization. ${ }^{14}$ This makes any comparison with many other TACE protocols difficult. Inherent limitations of this study include its retrospective nature as well as an unintentional selection bias during treatment allocation.

For a locoregional treatment approach to be successful, targeting as much of the tumor as possible is essential. Given that patients often present with an intermediate tumor burden, a single treatment modality may often be insufficient. Therefore, the argument for combining locoregional therapies to achieve complete tumor cell death cannot be overstated. The combination of TACE with RFA seems to efficiently and safely control tumor burden locally. More long-term and prospective studies are warranted to demonstrate an advantage in survival benefit of this combination therapy for commonly encountered medium-sized hepatocellular carcinomas.
DISCLOSURE J.-F.H.G.: Consultant-Biocompatibles, Bayer HealthCare, Guerbet, Nordion. Grant Support: Biocompatibles, Genentech, Bayer HealthCare, Philips Medical, Nordion, Context Vision, CeloNova, and RSNA. Founder and CEO-PreScience Labs, LLC.

\section{REFERENCES}

1. Altekruse SF, McGlynn KA, Reichman ME. Hepatocellular carcinoma incidence, mortality, and survival trends in the United States from 1975 to 2005. J Clin Oncol. 2009;27:1485-91.

2. Liapi E, Georgiades CC, Hong K, Geschwind JF. Transcatheter arterial chemoembolization: current technique and future promise. Tech Vasc Interv Radiol. 2007;10:2-11.

3. Llovet JM, Bru C, Bruix J. Prognosis of hepatocellular carcinoma: the BCLC staging classification. Semin Liver Dis. 1999;19:329-38.

4. Lencioni RA, Allgaier HP, Cioni D, et al. Small hepatocellular carcinoma in cirrhosis: randomized comparison of radio-frequency thermal ablation versus percutaneous ethanol injection. Radiology. 2003;228:235-40.

5. Lencioni R, Cioni D, Crocetti L, et al. Early-stage hepatocellular carcinoma in patients with cirrhosis: long-term results of percutaneous image-guided radiofrequency ablation. Radiology. 2005;234:961-7.

6. Rossi S. Percutaneous radiofrequency thermal ablation of nonresectable hepatocellular carcinoma after occlusion of tumor blood supply. Radiology. 2000; 217:119-26.

7. Kitamoto M, Imagawa $\mathrm{M}$, Yamada $\mathrm{H}$, et al. Radiofrequency ablation in the treatment of small hepatocellular carcinomas: comparison of the radiofrequency effect with and without chemoembolization. AJR Am J Roentgenol. 2003;181:997-1003.

8. Yamakado K, Nakatsuka A, Ohmori S, et al. Radiofrequency ablation combined with chemoembolization in hepatocellular carcinoma: treatment response based on tumor size and morphology. J Vasc Interv Radiol. 2002;13:1225-32.

9. Yamakado K, Nakatsuka A, Takaki H, et al. Subphrenic versus nonsubphrenic hepatocellular carcinoma: combined therapy with chemoembolization and radiofrequency ablation. AJR Am J Roentgenol. 2010;194:530-5.

10. Morimoto M, Numata K, Kondou M, et al. Midterm outcomes in patients with intermediate-sized hepatocellular carcinoma: a randomized controlled trial for determining the efficacy of radiofrequency ablation combined with transcatheter arterial chemoembolization. Cancer. 2010;116:5452-60.

11. Davis GL, Alter MJ, El-Serag $\mathrm{H}$ et al. Aging of gepatitis $\mathrm{C}$ virus (HCV)-infected persons in the United States: a multiple cohort model of HCV prevalence and disease progression. Gastroenterology. 2010; 38:513-21

12. Kawaoka T, Aikata H, Takaki S, et al. Transarterial infusion chemotherapy using cisplatin-lipiodol suspension with or without embolization for unresectable hepatocellular carcinoma. Cardiovasc Intervent Radiol. 2009;32:687-94.

13. Maeda N, Osuga K, Higashihara $\mathrm{H}$ et al. Transarterial chemoembolization with cisplatin as second-line treatment for hepatocellular carcinoma unresponsive to chemoembolization with epirubicin-lipiodol emulsion. Cardiovasc Intervent Radiol. 2011.

14. Liapi E, Geschwind JF. Transcatheter and ablative therapeutic approaches for solid malignancies. J Clin Oncol. 2007;25:978-86. 